\title{
RELAÇÃO ENTRE NÍVEIS DE RUÍDO, PRODUTIVIDADE E USO DE EPI EM UMA PEQUENA EMPRESA CALÇADISTA
}

\section{RELATIONSHIP BETWEEN NOISE LEVELS, PRODUCTIVITY AND USE OF PPE IN A SMALL FOOTWEAR COMPANY}

João Guilherme Marcato, Rafael Zampieri Krambek, Rafael Bellotti Moreno

Universidade Estadual Paulista - UNESP, Faculdade de Engenharia - FEB, Bauru, SP.

E-mail: ig marcato@hotmail.com; rapou2@hotmail.com; rflbmoreno@gmail.com

\begin{abstract}
RESUMO - Os efeitos do ruído sobre a saúde e a produtividade representam dilemas ambientais e ocupacionais para as organizações, podendo ser mitigados pela conscientização da importância e adoção de equipamentos de proteção individuais (EPI). Todavia, a análise dos níveis de ruído e o uso de tais equipamentos são negligenciados em micro e pequenas empresas (MPE). $O$ presente estudo buscou identificar a relação entre os níveis de ruído e produtividade em uma pequena empresa do setor calçadista, na qual se desenvolveram dois estudos de tempos: com e sem o uso de protetores auriculares. Os resultados demonstraram que os níveis de ruído têm efeito negativo sobre a produtividade e a existência de uma diferença real e significativa na produtividade dos operadores com e sem o uso de EPI.

Palavras-chave: ruído; produtividade; estudo de tempos.
\end{abstract}

\begin{abstract}
The effects of noise on health and productivity represent environmental and occupational issues for organizations, which can be mitigated by awareness of the importance and adoption of personal protective equipment (PPE). However, the analysis of noise levels and the use of the said equipment are neglected in micro and small enterprises (MSE's). The present study aimed to identify the relationship between noise levels and productivity in a small footwear company, in which two time studies were conducted: with and without the use of hearing protective devices. The result showed that noise levels have a negative effect on productivity and the existence of a real and significant difference in the operator's productivity with and without the use of PPE.
\end{abstract}

Keywords: noise; productivity; time study.

\section{INTRODUÇÃO}

No Brasil, há 6,4 milhões de empresas, dentre as quais $99 \%$ correspondem à micro e pequenas empresas (MPE), responsáveis por empregar 16,1 milhões de trabalhadores (52\%) no setor privado (SEBRAE, 2014). Todavia, segundo Couto (2006), em sua maioria, o uso de equipamentos de proteção individual (EPI) é negligenciado nessas empresas. Ainda conforme Couto (2006), o aumento da produção, pago sob a forma de incentivos salariais, faz com que os operadores exerçam suas atividades em ambientes extremamente insalubres, buscando auxílio médico somente após a manifestação de uma doença ocupacional grave. 
Tal insalubridade se manifesta por meio de um conjunto de fatores ambientais que afetam a qualidade de vida no trabalho (QVT), como temperatura, vibração, ventilação, iluminação e ruído (AKBARI et al., 2013). Este último, em especial, quando não controlado em ambientes fabris, eleva os índices de absenteísmo e de acidentes de trabalho (NOWEIR, 1984; DALTON; BEHM, 2007); promove o desenvolvimento de doenças ocupacionais, tais como: perda parcial ou total da audição; aumento da pressão arterial; estresse físico e mental; distúrbios psicológicos; náuseas; e cefaleias (VERMEER; PASCHIER, 2000; ATTARCHI et al., 2012; RUBIO-ROMERO et al., 2018); e diminui a produtividade (POJE et al., 2015; ALARJA; AWADALLAH, 2020).

Em relação à produtividade, é importante ressaltar que as metas de produção usalmente são determinadas em função das variáveis operacionais, desconsiderando, assim, as limitações físicas e mentais dos operadores (SOUZA et al., 2012). Nesse cenário, o emprego do estudo de tempos tem se tornado frequente em indústrias dos setores de manufatura e serviços a fim de utilizar eficientemente seus recursos e auferir vantagens competitivas (CHANDRA, 2013).

A literatura é rica em trabalhos que relacionam o estudo de tempos a ganhos produtivos e de capacidade por meio de reformulação de processos ou simulações computacionais, como é o caso, por exemplo, daqueles desenvolvidos por Burri Jr. e Helander (1991); Al-Saleh (2011); e Duran et al. (2015). Todavia, escassos são aqueles que avaliam o efeito do ruído sobre a produtividade em MPE. Dada a relevância do tema e a sua aparente lacuna no Estado da Arte, o presente trabalho busca responder ao seguinte problema de pesquisa: "Qual relação existe entre os níveis de ruído, a produtividade e o uso de EPI em MPE?"

Para tanto, desenvolveram-se dois estudos de tempos com todos os operadores de uma pequena empresa do setor calçadista: o primeiro, sem a utilização de protetores auriculares e o segundo, com a utilização deles. Os dados foram analisados com o auxílio do software IBM SPSS Statistics 21, com o qual se conduziram dois testes: o coeficiente de correlação de Spearman $\left(r_{s}\right)$, a fim de se verificar o impacto dos níveis de ruído sobre a produtividade; e o de Wilcoxon, com o intuito de se identificar diferenças significativas entre a produtividade dos operadores com e sem o uso de EPI.

Além dessa seção introdutória, a revisão da literatura é tecida na seção 2 . O método é definido na seção 3 , os resultados e discussões são apresentados na seção 4 e as considerações finais na seção 5, seguida pelas referências.

\section{REVISÃO DA LITERATURA}

Fundamentando este estudo, a literatura revisada contempla aspectos relacionados ao ruído e produtividade; ruído e qualidade de vida no trabalho e uso de EPI.

\subsection{Ruído e produtividade}

O ruído, caracterizado como um estímulo auditivo que não apresenta informações úteis para a execução de um conjunto de atividades, tem sido considerado um incômodo para a sociedade ao longo da história (KAMARULZAMAN et al., 2011). Conforme Dalton e Behm (2007), em ambientes industriais, este estímulo auditivo é responsável por causar distração e desmotivação, bem como elevar o estresse físico e mental dos trabalhadores.

Tal fato conduziu a realização de inúmeros estudos em médias e grandes empresas a fim de determinar o efeito da exposição prolongada ao ruído sobre a produtividade dos operadores (AKBARI et al., 2013; POJE et al., 2015; AL-ARJA; AWADALLAH, 2020).

O trabalho de Akbari et al. (2013), por exemplo, demonstrou a existência de uma correlação significativa e negativa ( $p$-valor $<0,05$ e $r=-0,178$ ) entre os níveis de ruído e a produtividade de trabalhadores de uma grande empresa montadora de veículos japonesa. Os autores concluíram que o ruído tem um impacto negativo sobre esta última, cuja melhoria deve se pautar na adoção de programas de controle e regulação do primeiro.

Corroborando com o exposto, o estudo desenvolvido por Poje et al. (2015) em uma madeireira alemã de médio porte concluiu que a produtividade diminui à medida que os níveis de ruído aumentam e que o tempo de exposição é essencial para analisar esta relação, resultado este reiterado por Al-Arja e Awadallah (2020) após desenvolverem um estudo de caso com 13 empresas moedoras de café na Jordânia.

A literatura também contempla pesquisas que relacionam ganhos de produtividade à melhoria de resultados oriundos de estudos de 
tempos por meio da mitigação de fatores relacionados ao ruído (SOUZA et al., 2012; NASSIRI et al., 2014). O estudo de Souza et al. (2012), por exemplo, demonstrou que adoção de um Programa de Conservação Auditiva (PCA) associado à conscientização dos operadores acerca da importância do uso de protetores auriculares diminuiu o tempo total de operação de uma empresa de corte florestal, promovendo, assim, um aumento de $5 \%$ na produtividade total da organização.

Nassiri et al. (2014) concluíram, por sua vez, que o controle do ruído em meios industriais afeta diretamente a produtividade, dado que diminui a distração do ambiente e, consequentemente, os erros, aumentando, logo, a velocidade e o volume das operações.

\subsection{Ruído e qualidade de vida no trabalho}

A existência de uma série de diferentes atividades laborais e condições humanas em um ambiente de trabalho que não correspondem às necessidades físicas e funcionais dos trabalhadores pode impactar negativamente $\mathrm{o}$ desempenho e a qualidade de vida deles, bem como trazer malefícios a sua saúde (NOWEIR, 1984).

Segundo Vermeer e Paschier (2000), o ruído é uma variável que pode prejudicar a QVT e a produtividade do trabalhador, configurando-se, assim, como um fator ambiental cujos efeitos devem ser conhecidos por todos os níveis organizacionais. No entanto, conforme Ganime et al. (2010) e Pommerrehn et al. (2016), sua avaliação é muitas vezes negligenciada por muitos empregadores e funcionários.

Como consequência, a exposição prolongada a ele, além de provocar zumbido, náuseas e perda de audição, contribui para o aumento do estresse ocupacional e o desenvolvimento de problemas auditivos, cardiovasculares, gastrointestinais, psíquicos, neurológicos e respiratórios (VERMEER; PASCHIER, 2000; ATTARCHI et al., 2012; RUBIOROMERO et al., 2018).

Attarchi et al. (2012), por exemplo, conduziram um estudo em uma empresa fabricante de borrachas a fim de verificar as relações entre os turnos de trabalho, a exposição ao ruído e a pressão arterial dos trabalhadores por meio da regressão logística. Os resultados foram significativos ( $p$-valor $<0,05$ ) e demonstraram que o trabalho em turnos e a exposição simultânea ao ruído têm efeito aditivo no desenvolvimento de problemas cardiovasculares, como a hipertensão.

Rubio-Romero et al. (2018), por sua vez, buscaram analisar os efeitos da exposição prolongada ao ruído sobre a audição de 115 trabalhadores de moinho de azeite entre 2003 e 2013. Os autores identificaram que houve um aumento significativo dos níveis de ruído aos quais os funcionários eram diariamente expostos durante o período considerado, cujos efeitos acarretaram no desenvolvimento de um conjunto de fatores que diminuem a qualidade de vida após a jornada de trabalho, tais como: cefaleia; tontura; zumbido nos ouvidos; elevação do nível de estresse; e, em casos extremos, perda total da audição.

\subsection{Ruído e o uso de EPI}

O conhecimento dos trabalhadores acerca dos riscos ocupacionais aos quais se sujeitam no desempenho de suas funções é em grande parte reduzido, fazendo com que eles não possuam consciência de que precisam de EPI específicos e adequados para realizá-las (ROCHA et al., 2014).

Filipi et al. (2014) afirmam que este processo de conscientização traz diversos benefícios para o empregado e para o empregador, em termos de eficiência, qualidade de vida, custos e redução dos acidentes de trabalho, bem como destacam que os protetores auriculares se configuram como EPI de baixo custo que podem alterar completamente a situação laboral de um ambiente marcado por elevados níveis de ruído.

Thepaksorn et al. (2018) conduziram um estudo com 540 trabalhadores de quatro empresas madeireiras da Tailândia a fim de compreender a relação entre a percepção do risco relacionado à exposição ao ruído e o uso de protetores auriculares. Os resultados demonstraram que os funcionários estão mais propensos a utlizarem estes EPI baseados na percepção do risco aos quais estão expostos, sendo esta oriunda de programas de treinamento e capacitação oferecidos pelas empresas analisadas.

Reiterando o exposto, Tinoco et al. (2019) concluíram que eficiência no uso destes protetores está diretamente relacionada à percepção dos operadores e da consciência dos mesmos em relação aos riscos no contexto do ruído. Segundo os autores, é fundamental que as organizações se comprometam tanto com o 
fornecimento quanto com a conscientização de seus funcionários em relação à importância do uso destes equipamentos.

\section{MÉTODO}

O presente estudo foi conduzido ao longo de todo o mês de outubro de 2019 em uma pequena empresa do setor calçadista localizada no município de Jahu (SP), caracterizado nacionalmente como um arranjo produtivo local voltado para a confecção de calçados femininos (ALBAGLI; BRITO, 2003). Esta emprega nove profissionais contratados, cada qual responsável por uma única etapa do processo produtivo da organização: dois para os processos de corte; cinco para montagem; e dois para limpeza.

O fluxo da cadeia produtiva parte dos processos de corte e logo se interrompe para um serviço terceirizado de pesponto; retornando a fábrica, atende aos processos de montagem e se finaliza nos processos de limpeza, que se encarregam também do encaixotamento dos produtos.

Logo, a primeira etapa do método adotado para este trabalho consistiu no mapeamento dos postos de trabalho ao longo do arranjo físico da empresa estudada, considerando o fluxo produtivo supracitado. Estes foram denominados como: Corte 1 (C1), referente ao processo de corte manual; Corte 2 ( $C 2)$, referente ao corte mecanizado; Montagem 1 (M1), colagem; Montagem 2 (M2), conformação; Montagem 3 (M3), montagem do bico; Montagem 4 (M4), montagem do traseiro; Montagem 5 (M5), solagem; Limpeza 1 (L1), limpeza e polimento; e Limpeza 2 (L2), embalagem e expedição. A Figura 1, abaixo, ilustra a disposição destes postos, bem como dos estoques e da sala de máquinas no layout da empresa estudada.

Figura 1. Mapeamento dos postos de trabalho.

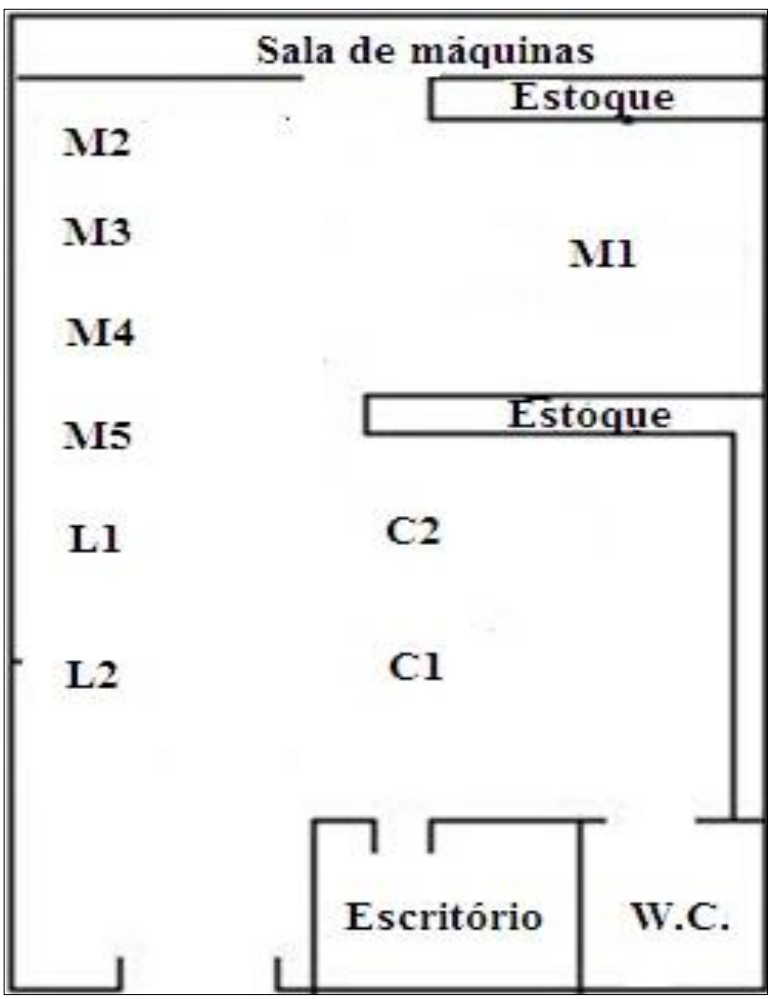

Fonte: Elaborada pelos autores (2019).

Uma vez finalizado o mapeamento, buscou-se caracterizar os funcionários. Para tanto, coletaram-se informações demográficas idade e gênero - e relacionadas ao trabalho experiência no setor calçadista exercendo a atividade desempenhada na empresa em estudo; desenvolvimento de alguma doença ocupacional em decorrência da exposição prolongada ao ruído, como perda parcial ou total da audição, estresse, naúseas e cefaleias, após a jornada de trabalho; e o uso de EPI na empresa analisada.

A etapa seguinte tratou de mensurar o nível de ruído para cada estação de trabalho analisada. Instalaram-se, em cada uma delas, 
decibelímetros digitais INS-1358 próximos aos ouvidos dos operadores e configurados com circuito de compensação " $A$ " e circuito de resposta lenta (SLOW), conforme estabelecido pelo Anexo I da Norma Regulamentadora 15 (NR 15) que versa sobre os limites de tolerância para ruído contínuo e intermitente no ambiente de trabalho (NR 15, 2019).

Seguindo o método proposto por Akbari et al. (2013), os níveis de ruído para cada posto foram coletados ao longo de uma jornada diária de trabalho de 8 horas por cinco dias, totalizando, assim, um período de 40 horas. O nível médio de ruído (NMR) para cada estação foi então calculado e comparado com aquele previsto pela NR 15, segundo a qual os níveis de ruído não devem exceder 85 decibéis $(\mathrm{dB})$ para uma exposição máxima de 8 horas por dia (NR 15, 2019).

O próximo passo consistiu em mensurar a produtividade de cada operador por meio da cronoanálise. Cada uma de suas respectivas atividades foram divididas em elementos a fim de serem submetidas ao estudo de tempos. 0 método empregado foi o da cronometragem contínua, caracterizado, conforme Barnes (1988) e Chandra (2013), pela manutenção do cronômetro em movimento ao longo de todo o estudo.

Realizaram-se dez medições para todas as operações. O elemento representativo da produtividade considerado foi a média dos tempos, expressa em segundos para a produção de um par de calçados. Tal estudo foi conduzido duas vezes no período vespertino da empresa em um mesmo dia, sendo que, na segunda, cada um dos operadores recebeu e utilizou um par de protetores auriculares.

A etapa final consistiu na análise estatística dos dados com o auxílio do software IBM SPSS Statistics 21 que se dividiu em três fases: a análise descritiva; a análise dos níveis de ruído sobre a produtividade; e a análise da produtividade com e sem o uso de EPI.

A análise descritiva tratou de caracterizar a amostra coletada, apresentando os dados referentes aos operadores, os níveis de ruído em cada estação de trabalho analisada e a produtividade de cada uma delas, expressa sob a forma de dois estudos de tempos: com e sem o uso de EPI.

A análise dos níveis de ruído sobre a produtividade, por sua vez, buscou analisar a relação entre estas variáveis por meio de um teste de correlação e sua significância estatística.

Tal teste visa verificar se as duas variáveis analisadas estão associadas e variam conjuntamente, valendo-se, para tanto, do coeficiente de correlação que varia de $-1 \mathrm{a}+1$, sendo que valores menores que zero representam correlação negativa. Dois coeficientes são contemplados pela literatura: o de Pearson $\left(r_{p}\right)$, que deve ser adotado quando a distribuição dos dados da amostra coletada é paramétrica, isto é, apresentam normalidade; e o de Spearman $\left(r_{s}\right)$, adotado quando esta é não paramétrica (ARTUSI; VERDERIO; MARUBINI, 2002).

Verificou-se a normalidade dos dados coletados por meio dos testes Shapiro-Wilk e Kolmogorov-Smirnov. P-valor superior a 0,05 indica normalidade (HAIR et al., 2014). Os resultados demonstraram que a amostra é não paramétrica ( $p$-valor $<0,05)$ para ambos os testes e, logo, decidiu-se pelo $r_{s}$.

A análise da produtividade com e sem o uso de EPI, por fim, buscou identificar a existência de uma diferença real e significativa entre a produtividade dos operadores com e sem o uso de protetores auriculares. Quando se pretende comparar médias obtidas de uma mesma amostra sob condições diferentes, como é o caso do presente estudo, e a variável analisada possui distribuição normal, recomendase a aplicação do teste "t" pareado (COELHO BARROS; MAZUCHELI, 2005).

Todavia, como a amostra coletada é não paramétrica, escolheu-se o teste de Wilcoxon que se pauta nas diferenças entre os escores das duas condições de comparação (escore Z) e permite que o valor exato da significância seja calculado com base na distribuição normal, fornecendo um p-valor correspondente a estatística bicaudal (Sig. (2-tailed)) (BARINGHAUS; GAIGALL, 2018).

\section{RESULTADOS E DISCUSSÕES}

Nesta seção, os resultados do estudo de campo serão apresentados e discutidos à luz do referencial teórico revisado. Para tanto, valer-seá da análise descritiva dos dados obtidos, seguida pelas análises estatísticas dos efeitos dos níveis de ruído sobre a produtividade; e da produtividade com e sem o uso de EPI.

\subsection{Análise descritiva dos dados}

Dos nove funcionários entrevistados, três eram do sexo feminino e seis do sexo masculino. 
A média das idades foi de 41 anos, com desviopadrão de 8,974, sendo a menor delas 32 anos e a maior, 58 anos.

Conforme Gomes et al. (2012), sob a perspectiva da Gestão do Conhecimento, a compreensão sistêmica das atividades em ambientes fabris se dá quando o operador possui experiência e domínio total de sua função. Logo, pode-se constatar que esta compreensão foi identificada na empresa em estudo, dado que o tempo médio de atuação no setor calçadista em uma atividade específica por seus funcionários foi de 16 anos com desvio de 4,051.

Estes afirmaram sofrer ou já terem sofrido as consequências da exposição ao ruído intermitente após o final da jornada de trabalho, tais como: cefaleia; tontura; zumbido nos ouvidos; e elevação do nível de estresse, reiterando, assim, os resultados dos estudos desenvolvidos por Vermeer e Paschier (2000), Attarchi et al. (2012) e Rubio-Romero et al. (2018), segundo os quais o ruído promove o desenvolvimento de doenças ocupacionais e diminui a qualidade de vida dos funcionários.

Todos também afirmaram que o uso de EPI na empresa estudada é totalmente neglicenciado, corroborando, assim, com a caracterização entre as MPE e o uso de tais equipamentos, discutida por Couto (2006). A Tabela 1, abaixo, apresenta o NMR para cada uma das estações de trabalho analisadas, bem como o NMR médio de todas elas.

Tabela 1. Nível médio de ruído auferido.

\begin{tabular}{c|c}
\hline $\begin{array}{c}\text { Estação de } \\
\text { trabalho }\end{array}$ & $\begin{array}{c}\text { NMR } \\
\text { (dB) }\end{array}$ \\
\hline $\mathrm{C} 1$ & 86,8 \\
\hline $\mathrm{C} 2$ & 89,2 \\
\hline $\mathrm{M} 1$ & 96,7 \\
\hline $\mathrm{M} 2$ & 102,9 \\
\hline $\mathrm{M} 3$ & 101,5 \\
\hline $\mathrm{M} 4$ & 97,4 \\
\hline $\mathrm{M} 5$ & 86,7 \\
\hline $\mathrm{L} 1$ & 88,2 \\
\hline $\mathrm{L} 2$ & 85,2 \\
\hline NMIR médio & 92,7 \\
\hline
\end{tabular}

Fonte: (Autores, 2019).

A análise dos resultados permite constatar que todos os postos de trabalho apresentam níveis de ruído superiores à $85 \mathrm{~dB}$, limite máximo previsto pela NR 15 (2019) para jornadas de trabalho de oito horas diárias. O menor NMR foi encontrado em L2 (85,2 dB) ao passo que o maior em M2 (102,5 dB). O NMR médio para todos os postos de trabalho foi de 92,7 dB, superior, também, ao limite previsto. Pode-se concluir, logo, que o ruído se configura como um fator ambiental que prejudica a QVT na empresa estudada.

A Tabela 2 apresenta a produtividade de cada um dos operadores, o desvio-padrão (desv) e o tempo total para concluir todas as operações para o estudo de tempos realizado sem o uso de EPI. 
Tabela 2. Produtividade sem o uso de EPI.

\begin{tabular}{c|c|c}
\hline $\begin{array}{c}\text { Estação de } \\
\text { trabalho }\end{array}$ & $\begin{array}{c}\text { Produtividade } \\
\text { (s) }\end{array}$ & desv \\
\hline C1 & 152,97 & 0,623 \\
\hline C2 & 11,48 & 0,411 \\
\hline M1 & 38,49 & 0,718 \\
\hline M2 & 99,76 & 0,895 \\
\hline M3 & 24,77 & 0,789 \\
\hline M4 & 5,37 & 0,218 \\
\hline M5 & 90,28 & 0,448 \\
\hline L1 & 50,75 & 0,229 \\
\hline L2 & 29,75 & 0,248 \\
\hline Total & 503,62 & - \\
\hline
\end{tabular}

Fonte: (Autores, 2019).

Os resultados demonstraram que 0 tempo necessário para finalizar todas as etapas do processo produtivo analisado sem o uso de qualquer EPI por parte dos operadores é de $503,62 \mathrm{~s}$.

Destaca-se que os maiores desviospadrão foram encontrados nos postos M1 $(0,789), M 2(0,895)$ e $M 3(0,789)$, justamente aqueles que, segundo a Tabela 1 , apresentam NMR superior ao NMR médio. Tal fato reforça a tese de Dalton e Behm (2007), indicando que o ruído pode influenciar a realização das atividades, pela promoção da distração do trabalhador.

A Tabela 3, por sua vez, apresenta a produtividade de cada um dos operadores, o desv e o tempo total para concluir todas as operações para o estudo de tempos realizado com o uso de EPI.

Tabela 3. Produtividade com o uso de EPI.

\begin{tabular}{c|c|c}
\hline $\begin{array}{c}\text { Estação de } \\
\text { trabalho }\end{array}$ & $\begin{array}{c}\text { Produtividade } \\
\text { (s) }\end{array}$ & desv \\
\hline C1 & 148,10 & 0,339 \\
\hline C2 & 9,96 & 0,189 \\
\hline M1 & 35,44 & 0,337 \\
\hline M2 & 94,90 & 0,485 \\
\hline M3 & 20,72 & 0,476 \\
\hline M4 & 4,40 & 0,109 \\
\hline M5 & 85,84 & 0,215 \\
\hline L1 & 49,94 & 0,187 \\
\hline L2 & 28,54 & 0,194 \\
\hline Total & 477,84 & - \\
\hline
\end{tabular}

Fonte: (Autores, 2019).

O tempo para concluir todas as etapas do processo produtivo com a utilização do protetor auricular pelos operadores foi de 477,84 s, representando uma redução de $25,78 \mathrm{~s}$ e um ganho de produtividade de $5,12 \%$ em relação ao estudo de tempos conduzido sem o uso de EPI. Este resultado vai ao encontro daquele encontrado por Souza et al. (2012) que, após sugerirem a adoção de protetores auriculares em uma empresa de corte florestal, obtiveram ganhos produtivos de $5 \%$.

Ressalta-se, também, que os desviospadrão de todas as atividades diminuíram com a adoção dos EPI supracitados, sendo que as reduções mais acentuadas foram encontradas naquelas estações de trabalho mais afetadas pelo ruído: M1 (de 0,718 para 0,337); M2 (0,895 para 0,485); e M3 (de 0,789 para 0,476).

Este resultado reitera as conclusões de Nassiri et al. (2014), segundo os quais o controle do ruído em meios industriais afeta diretamente a produtividade, dado que diminui os erros no ambiente de trabalho, auxiliando, assim, na padronização das atividades que passam a se desenvolver de maneira mais assertiva e constante. 
4.2. Análise do efeito dos níveis de ruído sobre a produtividade

Empregou-se o teste do coeficiente de correlação de Spearman $\left(r_{s}\right)$ para verificar o impacto dos níveis de ruído sobre a produtividade. P-valor inferior a 0,05 indica significância estatística do $\mathrm{r}_{\mathrm{s}}$ (ARTUSI; VERDERIO; MARUBINI, 2002).

Considerando o NMR e a produtividade de cada posto de trabalho com e sem o uso de EPI, o valor encontrado para $r_{s}$ foi de $-0,215 \mathrm{com}$ um $p$-valor igual a 0,002 (p-valor $<0,05$ ), indicando, assim, a existência de uma correlação negativa e significativa entre os níveis de ruído e produtividade, cuja probabilidade de tal associação ter acontecido ao acaso é inferior a $5 \%$.

A Figura 2 apresenta o gráfico de dispersão das variáveis analisadas, no qual se traçou também a curva linear entre elas.

Figura 2. Dispersão das variáveis.

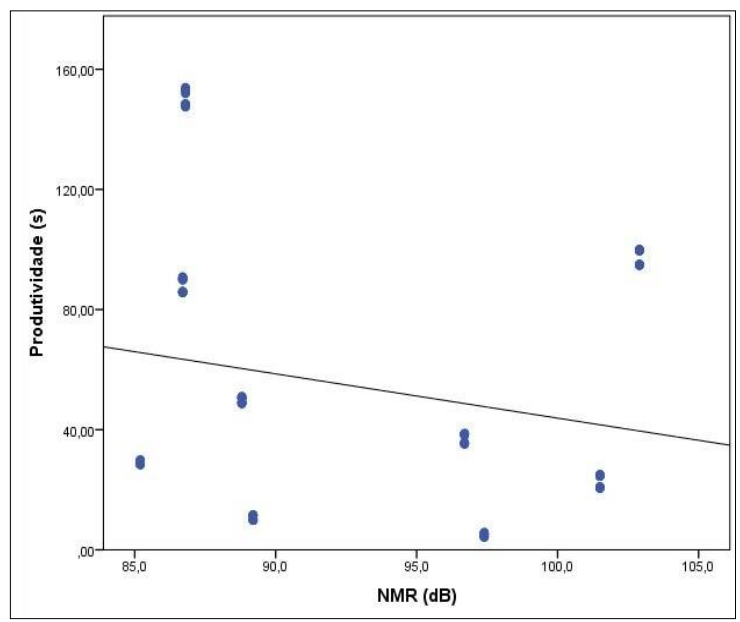

Fonte: (Autores, 2019).

A curva traçada é regida pela seguinte equação:

$$
Y=-1,47 \cdot X+191,31
$$

Onde $\mathrm{Y}$ representa a produtividade e $\mathrm{X}, \mathrm{O}$ NMR. As constantes numéricas $-1,47$ e 191,31 representam, respectivamente, os coeficientes angular (m) e linear (b) da curva e foram calculadas automaticamente pelo software estatístico empregado neste estudo.

Segundo Malhotra (2012), m representa a inclinação da curva em relação ao eixo horizontal ao passo que $b$ é a ordenada do ponto em que ela corta o eixo vertical. Caso $\mathrm{m}$ seja menor que zero, a declividade da reta é negativa, isto é, Y diminui a uma taxa constante na proporção em que $X$ aumenta.

Portanto, de acordo com (1), pode-se constatar que o NMR possui efeito negativo sobre a produtividade dos operadores, uma vez que, a cada dB acrescido, 1,47 s de produtividade se perde, e que esta seria igual a 191,31 s quando NMR fosse nulo.
A associação deste resultado com a significância estatística do teste de correlação permite concluir que há influência negativa do ruído sobre a produtividade na empresa estudada. Tal constatação reitera os resultados encontrados por Akbari et al. (2013), Poje et al. (2015) e Al-Arja e Awadallah (2020), que expressam que a produtividade diminui à medida que os níveis de ruído aumentam.

\subsection{Análise da produtividade com e sem o uso de EPI}

Empregou-se o teste de Wilcoxon para verificar a existência de diferenças significativas entre a produtividade dos operadores com e sem o uso de EPI. P-valor correspondente à estatística bicaudal (Sig. (2-tailed)) inferior a 0,05 indica que as diferenças são reais e significativas (BARINGHAUS; GAIGALL, 2018).

A Tabela 4, abaixo, apresenta os valores obtidos para o teste supracitado. 
Tabela 4. Estatísticas do teste Wilcoxon.

Fonte: (Autores, 2019)

\begin{tabular}{c|c}
\hline & com EPI-sem EPI \\
\hline Escore Z & $-8,239$ \\
\hline P-valor (Sig. (2-tailed)) & $9,275.10^{-6}$ \\
\hline
\end{tabular}

Constata-se, portanto, a existência de uma diferença real e significativa entre a produtividade dos operadores com e sem o uso de protetores auriculares na empresa estudada, dado que o $p$-valor é menor que 0,05 , indicando, assim, que a probabilidade de tais diferenças estarem relacionadas ao acaso é inferior a $5 \%$.

Este resultado vai ao encontro das conclusões do trabalho de Filipi et al. (2014) que indicam que $\mathrm{o}$ uso de protetores auriculares contribui para alterar a situação laboral de um ambiente marcado por elevados níveis de ruído, bem como ressalta o importante papel das organizações neste processo que, segundo Thepaksorn et al. (2018) e Tinoco et al. (2019), devem se comprometer a fornecer tais equipamentos e conscientizar seus funcionários a respeito da importância de seu uso.

\section{CONSIDERAÇÕES FINAIS}

O trabalho realizado buscou responder ao seguinte problema de pesquisa: "Qual relação existe entre os níveis de ruído, a produtividade e o uso de EPI em MPE?" Para tanto, desenvolveram-se dois estudos de tempos - com e sem o uso de EPI - em uma pequena empresa do setor calçadista, na qual o uso destes equipamentos é negligenciado e os níveis de ruído ultrapassam o limite máximo (85 dB) previsto pela NR 15 para uma jornada de trabalho diária de oito horas.

Os resultados demonstraram que os níveis de ruído têm efeito negativo sobre a produtividade ( $p$-valor $<0,05$ e $r_{s}=-0,215$ ) e a existência de uma diferença real e significativa ( $p$ valor $<0,05$ ) na produtividade dos operadores com e sem o uso de protetores auriculares em um ambiente caracterizado pelo ruído.

Este estudo contribui cientificamente ao ampliar a discussão acerca da importância de se avaliar a relação entre ruído e produtividade em MPE, analisando ganhos de produtividade, associados à cronoanálise, oriundos da correção específica do problema do ruído no ambiente industrial.

O trabalho contribui gerencialmente ao demonstrar a importância das organizações oferecerem as melhores condições possíveis de trabalho aos seus funcionários, dado que as condições do ambiente impactam diretamente a produtividade e a QVT do trabalhador. Tal fato se faz ainda mais relevante em MPE, nas quais o uso de EPI é, em sua maioria, negligenciado.

Nesse contexto, a conscientização dos gestores destas empresas de que é possível auferir ganhos de produtividade e melhorar a QVT por meio do desenvolvimento de um programa de controle e regulação dos níveis de ruído, cuja avaliação deve se dar de forma periódica, configura-se como uma forma de se mitigar os efeitos negativos oriundos da exposição prolongada a este fator ambiental.

Além disso, devem-se fornecer protetores auriculares a seus funcionários e desenvolver junto a eles programas de conscientização acerca da importância de se utilizar EPI e dos riscos aos quais estes estarão sujeitos caso desenvolvam suas atividades sem o uso deles.

É necessário ressaltar, contudo, que se considerou uma amostra pouco significativa de MPE do setor calçadista, uma vez que se analisou somente uma empresa, constituída por nove funcionários, e que se deve ter cuidado ao generalizar os resultados obtidos. Apesar disso, pode-se considerá-la aceitável, dada a natureza do trabalho.

Futuros estudos devem abarcar em seu escopo uma amostra maior de MPE tanto do setor calçadista quanto de outros segmentos, bem como realizar uma avaliação ergonômica do trabalho (AET) a fim de verificar a existência de outros fatores ambientais que, além do ruído, podem afetar a produtividade dos operadores, tais quais a iluminação e a temperatura.

Novos estudos devem também verificar se há influência de variáveis demográficas, como idade e gênero, e do tempo de experiência exercendo uma determinada função pelos operadores sobre a produtividade.

\section{REFERÊNCIAS}

AKBARI, J.; DEHGHAN, H.; AZMOON, H.; FOROUHARMAJD, F. Relationship between Light and Noise Levels and Productivity of the Occupants in Automotive Assembly Industry. 
Journal of Environmental and Public Health, 2013. https://doi.org/10.1155/2013/527078

AL-ARJA, O.; AWADALLAH, T. S. Assessment of occupational noise exposure in coffee grinding shops. Applied Acoustics, v. 158, 2020. https://doi.org/10.1016/j.apacoust.2019.107047

ALBAGLI, S.; BRITO, J. Glossário de arranjos e sistemas produtivos e inovativos locais. Rio de Janeiro: REDESIST/IE-RJ, 2003.

AL-SALEH, K. S. Productivity improvement of a motor vehicle inspection station using motion and time study techniques. Journal of King Saud University - Engineering Sciences, v. 23, n. 1, p. 33-41, 2011. https://doi.org/10.1016/j.jksues.2010.01.001

ARTUSI, R.; VERDERIO, P.; MARUBINI, E. BravaisPearson and Spearman correlation coefficients: meaning, test of hypothesis and confidence interval. International Journal of Biological Markers, v. 17, n. 2, p. 148-151, 2002. https://doi.org/10.1177/172460080201700213

ATTARCHI, M.; DEHGHAN, F.; SAFAKHAH, F.; NOJOMI, M.; MOHAMMADI, S. Effect of Exposure to Occupational Noise and Shift Working on Blood Pressure in Rubber Manufacturing Company Workers. Industrial Health, v. 50, p. 205-213, 2012. https://doi.org/10.2486/indhealth.MS1321

BARINGHAUS, L.; GAIGALL, D. Efficiency comparison of the Wilcoxon tests in paired and independent survey samples. Metrika, v. 81, p. 891-890, 2018. https://doi.org/10.1007/s00184$\underline{018-0661-4}$

BARNES, R. M. Estudo de movimentos e tempos: projeto e medida do trabalho. São Paulo: Edgar Blücher, 1988.

BURRI JR., G. J.; HELANDER, M. G. A field study of productivity improvement in the manufacturing of circuit boards. International Journal of Industrial Ergonomics, v. 7, n. 3, p. 207-215, $1991 . \quad$ https://doi.org/10.1016/01698141(91)90004-6

CHANDRA, P. V. An effort to apply work and time study techniques in a manufacturing unit for enhancing productivity. International Journal of
Innovative Research in Science, Engineering and Technology, v. 2, n. 8, 2013.

COELHO BARROS, E. A.; MAZUCHELI, J. Um estudo sobre o tamanho e poder dos testes tStudent e Wilcoxon. Acta Scientarium. Techonology, v. 27, n. 1, p. 23-32, 2005. https://doi.org/10.4025/actascitechnol.v27i1.149 $\underline{5}$

COUTO, H. A. Índice TOR-TOM: Indicador ergonômico da eficácia de pausas e outros mecanismos de regulação. Belo Horizonte: Ergo, 2006.

DALTON, B. H.; BEHM, D. G. Effects of noise and music on human and task performance: $A$ systematic review. Occupational Ergonomics, v. 7, p. 143-152, 2007.

DURAN, C.; CETINDERE, A.; AKSU, Y. E. Productivity Improvement by Work and Time Study Technique for Earth Energy-glass Manufacturing Company. Procedia Economics Finance, v. 26, p. 109-113, 2015. https://doi.org/10.1016/S2212-5671(15)00887-4

FILIPI, A. P.; SILVA , J. R. M; TRUGILHO, P. F.; FIEDLER , N. C.; RABELO, G. F.; BOTREL, D. A. Assessment of noise in furniture factories. CERNE, v. 20, n. 4, 2014. https://doi.org/10.1590/0104776020142004959

GANIME, J. P.; SILVA, L. A.; ROBAZZI, M. L. C. C.; VALENZUELA, S. S.; FALEIRO, S. A. El ruido como uno de los riegos ocupacionales: una revision de la literatura. Enfermeria Global, v. 19, 2010. https://doi.org/10.4321/S1695$\underline{61412010000200020}$

GOMES, A. L.; GOMES, M. L.; FERNANDES, E. S. G.; SILVA NETO, R. ; APRIGIO, A. S. Gestão do conhecimento nas organizações: a importância do ciclo do conhecimento. In: ENCONTRO NACIONAL DE ENGENHARIA DE PRODUÇÃO, 32., 2012, Bento Gonçalves, RS. Anais [...]. Bento Gonçalves, 2012.

HAIR, J. F.; BLACK, W. C.; BABIN, B. J.; ANDERSON, R. E. Multivariate Data Analysis. 7. ed. [São Paulo]: Pearson Education Limited, 2014.

KAMARULZAMAN, N.; SALEH, A. A.; HASHIM, S. Z.; HASHIM, H.; ABDUL-GHANI, A. A. An Overview 
of the Influence of Physical Office Environments towards Employees. Procedia Engineering, v. 20, p. 262-268, 2011. https://doi.org/10.1016/j.proeng.2011.11.164

MALHOTRA, N. Pesquisa de Marketing: uma orientação aplicada. 6. ed. Porto Alegre: Bookman, 2012.

NASSIRI, P.; MONAZZAM, M. R.; ASGHARI, M.; ZAKERIAN, S. A.; DEHGHAN, S. M.; FOLLADI, B.; $A Z A M, K$. The interactive effect of industrial noise type, level and frequency characteristics on occupational skills. Performance Enhancement \& Health, v. 3, n. 2, p. 61-65, 2014. https://doi.org/10.1016/i.peh.2015.01.001

NORMA REGULAMENTADORA 15 (NR 15) Atividades e Operações Insalubres, 2019. Disponível em: https://enit.trabalho.gov.br/portal/images/Arqui vos SST/SST NR/NR-15-atualizada-2019.pdf.

Acesso em: 01 set. 2019.

NOWEIR, M. H. Noise exposure as related to productivity, disciplinary actions, absenteeism, and accidents among textile workers. Journal of Safety Research, v. 15, n. 4, p. 163-174, 1984. https://doi.org/10.1016/0022-4375(84)90048-3

POJE, A.; SPINELLI, R.; MAGAGNOTTI, N.; MIHELIC, M. Exposure to noise in wood chipping operations under the conditions of agro-forestry. International Journal of Industrial Ergonomics, v. 50, p. 151-157, 2015. https://doi.org/10.1016/j.ergon.2015.08.006

POMMEREHN, J.; FILHA, V. A. V. S.; MIOLO, S. B.; FEDOSSE, E. Noise and quality of life in the perspective of gas station workers. Revista CEFAC, v. 2, n. 18, p. 377-384, 2016. https://doi.org/10.1590/1982-

$\underline{0216201618213515}$

ROCHA, L. P.; VAZ, M. R. C.; ALMEIDA, M. C. V.; BONOW, C. A.; SILVA, M. S.; COSTA,V .Z. Use of personal protective equipment by gas stations workers: a nursing contribution. Contexto Enferm, v. 1, n. 23, p. 193-202, 2014. https://doi.org/10.1590/S0104$\underline{07072014000100023}$

RUBIO-ROMERO, J. C.; CARILLO-CASTRILLO, J. A.; SORRIANO-SERRANO, M.; GALINDO-REYES, F.; DE
LA VARGA-SALTO, J. A longitudinal study of noise exposure and its effects on the hearing of olive oil mill workers. International Journal of Industrial Ergonomics, v. 67, p. 60-66, 2018. https://doi.org/10.1016/i.ergon.2018.04.003

SEBRAE - Serviço Brasileiro de Apoio às Micro e Pequenas Empresas. As micro e pequenas empresas na exportação brasileira. Brasília: SEBRAE, 2014. Disponível em: https://www.sebrae.com.br/Sebrae/Portal\%20Se brae/Anexos/As\%20MPE\%20na\%20expota\%c3\%a 7\%c3\%a30\%20brasileira estados 2012.pdf.

Acesso em: 12 ago. 2019.

SOUZA, A. P.; MINETTE, L. J.; SANCHES. A. L.; SILVA, E. P.; RODRIGUES, V. A.; OLIVEIRA, L. A. Ergonomic factors and production target evaluation in eucalyptus timber harvesting operations in mountainous terrains. Work, v.41, p. 4957-4962, 2012.

THEPAKSORN, P.; SIRIWONG, W.; NEITZEL, R. L.; SOMRONGTHONG, R.; TECHASRIVICHIEN, T. Relationship Between Noise-Related Risk Perception, Knowledge, and the Use of Hearing Protection Devices Among Rubber Wood Sawmill Workers. Safety and Health at Work, v. 9, p. 2529, 2018. https://doi.org/10.1016/j.shaw.2017.06.002

TINOCO, H. C.; LIMA, G. B. A.; SANT'ANNA, A. P.; GOMES, C. F. S.; SANTOS, J. A. N. Risk perception in the use of personal protective equipment against noise-induced hearing loss. Gestão \& Produção, v. 26, n. 1, 2019.

VERMEER, W.; PASSCHIER, W. F. Noise Exposure and Public Health. JSTOR - Environment Health Perspectives, n. 108, p. 123-131, 2000. https://doi.org/10.1289/ehp.00108s1123 